BMJ Open Sport \& Exercise Medicine

\title{
Reliability of the Shoulder Symptom Modification Procedure and association of within-session and between-session changes with functional outcomes
}

\author{
Adam Meakins, ${ }^{1}$ Stephen May, ${ }^{2}$ Chris Littlewood ${ }^{3}$
}

\begin{abstract}
To cite: Meakins A, May S, Littlewood C. Reliability of the Shoulder Symptom Modification Procedure and association of within-session and between-session changes with functional outcomes. BMJ Open Sport \& Exercise Medicine 2018;4:e000342. doi:10.1136/ bmjsem-2018-000342
\end{abstract}

- Additional material is published online only. To view please visit the journal online (http://dx.doi.org/10.1136/ bmjsem-2018-000342).

Accepted 5 March 2018

\section{Check for updates}

${ }^{1}$ Physiotherapy Department, Spire Bushey Hospital, Bushey, UK

${ }^{2}$ Faculty of Health and Wellbeing, Sheffield Hallam University,

Sheffield, UK

${ }^{3}$ Keele Clinical Trials Unit, Keele University, Staffordshire, UK

\section{Correspondence to}

Adam Meakins; adammeakins@ hotmail.com

\section{ABSTRACT}

Background Despite being a common problem, there is considerable diagnostic uncertainty with regard to shoulder pain. This uncertainty relates to the reliability and validity of current examination tests. The Shoulder Symptom Modification Procedure (SSMP) has been proposed as an alternative to existing approaches. Objective To evaluate interclinician reliability of the SSMP and the association of within-session and betweensession changes on clinical outcome at 1 week, and at 1 and 3 months.

Design A single-centre reliability study, with prospective follow-up.

Methods Twenty-six patients with shoulder pain were recruited. Following an initial SSMP-based examination, a second examination was performed by a second physiotherapist, blinded to the results of the first examination. Clinical outcome data were completed after 1 week, 1 month and 3 months via theNumeric Pain Rating Scale and the Shoulder PainandDisability Index. Reliability was evaluated using kappa and associations were evaluated using Spearman's r.

Results Inter-rater reliability of the SSMP was moderate $(\kappa=0.47)$. Association of within-session changes ranged from fair to poor in the short term $(r=0.24-0.01)$ to poor in the mid-term $(r=-0.03)$. The association of betweensession changes ranged from substantial to moderate in the short term $(r=0.74-0.47)$ but slight in the mid-term $(r=0.22)$.

Conclusions Based on this study, we cannot recommend the SSMP as a reliable tool for physical examination of patients with shoulder pain. The importance of within-session and between-session changes remains uncertain.

\section{INTRODUCTION}

Musculoskeletal conditions are now ranked as the second highest cause of number of years lived with disability. ${ }^{1}$ After low back and neck pain, shoulder pain is the third most common cause for musculoskeletal consultations, with up to $20 \%$ of people reporting a shoulder-related issue at any one time. ${ }^{2}$ A large percentage of these shoulder complaints do not recover spontaneously,

\section{What are the new findings?}

- The Shoulder Symptom Modification Procedure (SSMP) demonstrates moderate inter-rater reliability

- The importance of creating within-session changes in pain on outcome remains uncertain.

- Between-session changes in pain seem more strongly associated with outcome in the short term, but this diminishes in the mid-term.

How might it impact on clinical practice in the near future?

- There is insufficient evidence to recommend the SSMP as a reliable or validated evidence tool for physical examination of patients with shoulder problems.

with approximately $50 \%$ reporting persistent problems 6 months after onset and $40 \%$ reporting incomplete recovery at 1 year. ${ }^{2}$

Although there is considerable diagnostic uncertainty, the structures within the subacromial space such as the bursa and rotator cuff tendons are thought to be the most common causes of shoulder pain. ${ }^{3}$ However, despite such commonality and global burden, these disorders are poorly understood and poorly managed. ${ }^{4}$ The main reason for this could be the considerable diagnostic uncertainty, which is related to the limitations of the examination tests, in terms of reliability and sensitivity/specificity, used to diagnose pathology and inform treatment selection. ${ }^{5-7}$

Some of the limitations associated with current approaches to the physical examination of the shoulder were highlighted by May et $a \tilde{\varphi}$ in their systematic review, which concluded there is no consistent evidence that any physical examination test used in the assessment of the shoulder demonstrates acceptable levels of reliability. These findings were further supported by Hanchard et $a l^{7}$, who 
systematically reviewed 33 studies involving 3852patients that investigated the reliability of orthopaedic tests that were designed to identify lesions of the subacromial tendons, bursa and labrum. They found extreme diversity in the interpretation and performance of all these tests and insufficient evidence that current clinical testing is able to identify these issues reliably.

In response to the limitations associated with the current orthopaedic physical examination, a potential enhancement to the physical examination of the shoulder, the Shoulder Symptom Modification Procedure (SSMP) (https://www.londonshoulderclinic.com/ wp-content/uploads/2016/08/SSMPv6-2016.pdf), has been proposed with the aim of helping clinicians guide treatment without assumption about the underlying pathology. ${ }^{3}$ The SSMP is a series of standardised tests that attempt to reposition structures, facilitate movement or neuromodulate symptoms thought to arise from the shoulder. The aim of the SSMP is to identify whether such techniques can reduce pain associated with movement. It is hypothesised that these "within-session changes' (changes that occur during the examination) can be useful to help guide therapists to choose appropriate treatments and might help to predict those who might respond to physiotherapy. ${ }^{3}$ The predictive validity of a within-session and a between-session change has been explored in a number of regions, including the neck, ${ }^{8}$ shoulder, ${ }^{9}$ back $^{10}$ and hip. ${ }^{11}$

The SSMP is performed in a sequential format through four key areas: thoracic repositioning, scapula facilitation, humeral head procedures and neuromodulatory techniques. ${ }^{3}$ Thoracic repositioning tests are conducted first to see if this reduces or resolves a painful arm or shoulder movement. If not the SSMP moves onto a number of scapula-based repositioning and facilitation tests. If these do not resolve or reduce the painful movement, then the SSMP moves onto a series of humeral head techniques done in standing, sitting or supine. Finally if none of these procedures have affected patients' symptoms, a series of neuromodulatory techniques such as cervical distractions or mobilisation with movement, soft tissue massage, and taping techniques are attempted.

The SSMP has been widely adopted by many physiotherapists despite limited published evidence of its reliability or predictive value of the approach. ${ }^{12} 13$ Therefore, the purposes of this study were to (1) evaluate interclinician agreement of the SSMP (primary goal) and(2) evaluate the association of any initial within-session and between-session changes in pain on patient selfreport of pain and disability at the first follow-up session and at 1 and 3 months (secondary goal).

\section{METHODS}

\section{Design}

This is a single-centre reliability study, with prospective follow-up, based in a private hospital in Hertfordshire, UK. The manuscript is reported with reference to Quality Appraisal of Diagnostic Reliability checklist.

\section{Recruitment}

All patients over 18 years old with shoulder and/or upper arm pain who were referred for physiotherapy by their general practitioner and orthopaedic consultant or by self-referral were invited to participate in this study when they booked their appointment. Patients were informed of the study and its purpose, and all were given or posted a written information sheet outlining the study aims when making their first appointment. On average patients had between 1 and 7 days from making their booking to their first appointment, allowing them time to ask questions or withdraw from the study if they wished.

To be included all patients had to report and demonstrate, during initial examination by the lead author, shoulder and/or upper arm pain on elevation during flexion, scaption or abduction. They also had to report pain with or without weakness on isometric resisted testing into external rotation and/or abduction.

Patients with painful shoulder or upper arm pain that had significant signs of passive stiffness were excluded. We judged this to be anything more than a $25 \%$ loss of passive movement into external rotation and/or elevation when compared with the contralateral side. We also excluded any painful shoulder or upper arm that had signs of cervical spine involvement, such as pain reproduced in the shoulder or arm on cervical movement or axial compression, and/or if they had any positive distal neurological signs or symptoms on examination. Finally we excluded any patients with a history of shoulder surgery or gross orthopaedic trauma, such as dislocation or fracture, within the past 12 months.

\section{Clinicians}

All patients were first assessed by the lead author, a qualified physiotherapist with experience on treating patients with shoulder pain for over 15 years, who had received previous training in the use of the SSMP. The second assessors in this study comprised eight other qualified physiotherapists who worked with the lead author within the same physiotherapy department. Their clinical experience ranged from 3 to 9 years, and their understanding and exposure of the SSMP were variable. Two of the other assessors had completed SSMP workshops, three had read the SSMP papers, and three were unaware of the SSMP prior to participating in the study.

To ensure a comparable level of understanding and standardisation in the application of the SSMP, a 3-hourinservice training programme was attended by all the physiotherapists who would be involved prior to the start of the study. The lead author demonstrated the SSMP and ensured all other physiotherapists were consistent and confident in the procedures.

\section{Procedure}

Once informed written consent was gained, the participants completed the baseline Shoulder PainandDisability Index (SPADI). The SPADI is a self-report measure specifically developed to evaluate pain and function in 
patients with shoulder pathology. ${ }^{14} 15$ It is a commonly used measurethat has been validated for use in this patient population, and a minimally clinically important change of 10 points has been identified. The SPADI includes 13 items divided into twosubscales: pain (five items)and disability (eight items). The responses are indicated on a visual analogue scale, where $0=$ no pain/ no difficulty and $10=$ worst imaginable pain/so difficult it requires help. The items are summed and converted to a total score out of 100.

Following completion of the SPADI, the patient was then asked to demonstrate their most painful movement or activity and report their level of pain on a 0-10Numeric Pain Rating Scale (NPRS). The SSMP was then performed as described. The patient's response or lack of response to the SSMP was recorded on a standardised SSMP recording form (online supplementary appendix 1) and placed into a sealed envelope. A positive response to an SSMP test was classified as a complete resolution of symptoms or a reduction in the NPRS of 4 or more points to reflect prior guidance.

To reduce the risk of tissue irritation and an order effect, on completion of the SSMP examination by the first physiotherapist, the patient was asked to sit quietly in the assessment room for at least $10 \mathrm{~min}$, prior to the subsequent application of the SSMP by the second physiotherapist. Selection of the second physiotherapist was opportunistic according to current availability. The second assessor was not informed of the first assessor's results, only which side was to be assessed. The patients were also instructed not to discuss or inform the second therapist of what had or had not occurred previously with the first assessment. After $10 \mathrm{~min}$ had passed, the second therapist then performed the SSMP and recorded their findings on the same standardised form, and placed this into a second sealed envelope. Both sealed envelopes where then passed to a non-clinical administrator unaware of the SSMP and the data logged and recorded onto a secure spreadsheet to which the assessors had no access.

The patient's treatment then commenced with the lead author as usual. All patients were followed up and assessed a week later, and second NPRS and SPADI scores were obtained. Follow-up treatments after this session were dependent on symptoms, availability and need. NPRS and SPADI scoreswere further obtained at 1 month and 3 months after initial assessment.

\section{Data analysis}

Demographic details of the participants, including ageand duration of symptoms, are presented descriptively. Inter-rater agreement was analysed via SPSS using the kappa statistic. ${ }^{17}$ Kappa is a measure of chance-corrected agreement with the following interpretations being suggested: $\leq 0=$ poor, $0.01-0.20=$ slight, $0.21-0.40=$ fair, $0.41-0.60=$ moderate, $\quad 0.61-0.80=$ substantialand $\quad 0.81-$ $1.0=$ almost perfect. ${ }^{16}{ }^{17}$ A 95\% CI for kappa was obtained through bootstrapping (1000 samples).

\begin{tabular}{|ll|}
\hline $\begin{array}{l}\text { Table } 1 \\
(\mathrm{n}=26)\end{array}$ & Demographic description of the participants \\
\hline Mean age in years (SD) & $52.4(10.5)$ \\
\hline Gender (male/female) & $13 / 13$ \\
\hline Affected shoulder (right/left) & $11 / 15$ \\
\hline Dominant arm (right/left) & $22 / 4$ \\
\hline Duration of symptoms in months (SD) & $4.6(3.4)$ \\
\hline Baseline NPRS (SD) & $6.6(1.4)$ \\
\hline Post-SSMP NPRS (SD) & $2.3(1.5)$ \\
\hline Baseline SPADI (SD) & $46.5(14.3)$ \\
\hline
\end{tabular}

NPRS, Numeric Pain Rating Scale; SPADI, Shoulder Pain and Disability Index; SSMP, Shoulder Symptom Modification Procedure.

To reflect the data, the correlation between the degree of change (post-SSMP NPRS minus pre-SSMP NPRS) on the NPRS and the SPADI (SPADI at first follow-up session, 1 and 3 months minus SPADI at baseline) was analysed using the non-parametric Spearman's rank correlation coefficient to evaluate the impact of within-session change, and repeated using the degree of change over 1 week on the NPRS (NPRS at 1 week minus pre-SSMP NPRS) to evaluate the impact of between-session change.

\section{Sample size calculation}

Sample size was determined based on the primary goal of the study, which was to evaluate inter-rater reliability (two raters) with regard to the categories of the SSMP. We assumed a proportion of positive findings of $50 \%$, power of $80 \%$ and a (two-sided) significance level of $5 \%$, and hence aimed to recruit 32 participants. ${ }^{17}$

\section{RESULTS}

A total of 26 patients were recruited (table 1).

Of 26 patients, all reported abduction as their most painful shoulder movement. Twenty-five patients reported complete or partial response to the SSMP, based on the assessment by therapist 1 . Four patients reported complete response (NPRS $=0$ post-SSMP), with 21 reporting partial response. Of the 21 partial responders, two patients did not report change on the NPRS of greater than 4 points so were classified as overall non-responders. One patient reported no change in response to the SSMP. Hence, in total, 23 of 26 patients responded to initial SSMP procedures.

\section{Primary goal}

For therapist 1, response to SSMP was most commonly in relation to scapula repositioning $(n=10)$, followed by humeral head procedures $(n=8)$, thoracic repositioning $(n=2)$, neuromodulation $(n=2)$ and finally correction of scapula winging $(n=1)$. For the second therapists, response to SSMP was similar and most commonly in relation to scapula repositioning $(n=10)$, followed by humeral head procedures $(n=8)$, thoracic repositioning 
Table 2 Patient-reported outcome data

\begin{tabular}{lcccc}
\hline & Baseline & 1week & 1month & 3months \\
\hline Numeric Pain Rating Scale & 6.6 (SD 1.4) & $6.2($ SD 1.7) & 4.3 (SD 2.0) & 3.2 (SD 1.6) \\
Shoulder Pain and Disability Index & 46.5 (SD 14.3) & 39.6 (SD 14.9) & 28.8 (SD 17.0) & 21.4 (SD 14.7) \\
\hline
\end{tabular}

$(n=1)$, neuromodulation $(n=2)$, and correction of scapula winging $(n=1)$.

The physiotherapists agreed on 15/26 (57.7\%) of cases. Inter-rater agreement for the SSMP was moderate $(\mathrm{k}=0.47 ; 95 \% \mathrm{CI} 0.20$ to 0.71$)$.

\section{Secondary goal}

Patient-reported outcomes were reported at 1 week, 1 month and 3 months (table 2). On average, patients reported improvement from baseline to 3 months. The reduction in NPRS (6.6(SD 1.4) to 2.3(SD 1.5)) reported in response to the SSMP on day 1 was not maintained at 1 week (6.2 (SD 1.7)), 1 month (4.3 (SD 2.0)) or 3 months (3.2 (SD 1.6)).

The association between the amount of change on the NPRS from pre-SSMP to post-SSMP testing and the amount of change from baseline to 1 week on the SPADI ( $r=0.24 ; 95 \%$ CI-0.25 to 0.63 ) was fair, from baseline to 1 month on the SPADI $(r=0.01 ; 95 \%$ CI-0.43 to 0.49$)$ was poor, and from baseline to 3 months on the SPADI $(\mathrm{r}=-0.03$; $95 \% \mathrm{CI}-0.54$ to 0.63$)$ was poor in a negative direction.

The association between the amount of change on the NPRS from pre-SSMP (baseline) testing to week 1 and the amount of change from baseline to 1 week on the SPADI ( $r=0.74 ; 95 \%$ CI0.42 to 0.91$)$ was substantial, from baseline to 1 month on the SPADI ( $r=0.47 ; 95 \%$ CI0.01 to 0.82 ) was moderate, and from baseline to 3 months on the SPADI ( $r=0.22 ; 95 \%$ CI-0.37 to 0.76 ) was fair.

\section{DISCUSSION}

The data from this study suggest that the SSMP demonstrates moderate interclinician agreement $(\kappa=0.47)$. It has previously been suggested that kappa levels should be greater than 0.75 for a test to be of clinical utility. ${ }^{5} 18$ Hence, based on the findings of this study, there is insufficient evidence to recommend the SSMP as a reliable tool for physical examination of patients with shoulder problems.

With regard to the secondary goal, based on the findings of this study, within-session change in patients' self-report of pain secondary to application of the SSMP is not well associated with patient self-report of pain and disability over 1 week, 1 month or 3 months. Betweensession change, over 1 week, in patient self-report of pain secondary to application of the SSMP is substantially associated with patient self-report of pain and disability over 1 week, moderately associated at 1 month and only slightly associated by 3 months.

To date, two previous studies have evaluated the interclinician agreement of the SSMP; one study reported substantial levels of reliability, ${ }^{13}$ whereas the other study reported variable levels of reliability. ${ }^{12}$ The variability in findings might, in part, be explained by the different research designs. Bahat and Kerner ${ }^{12}$ evaluated reliability by asking two physiotherapists to examine the patient, whereas Lewis $e t a l^{13}$ evaluated reliability by video recording one physiotherapist examining the patient while other physiotherapists recorded their judgement about the response. This current study adopted a similar method to Bahat and Kerner ${ }^{12}$; this method has the advantage of mimicking real-world practice but with the risk that the examination of the patient by the first physiotherapist might change the presentation such that the second physiotherapist is examining a different clinical presentation, which hampers any judgement about reliability. In contrast, one of the advantages of the method used by Lewis $e t a l^{13}$ is that the judgements of a larger number of physiotherapists can be more easily garnered but with the risk that the process artificially standardises the examination process, which might erroneously elevate levels of reliability. A number of approaches to evaluating reliability have previously been adopted and there is no definitive guidance regarding the optimal approach. ${ }^{5}$ However, it seems appropriate to be mindful that the different reliability designs adopted might explain some of the variance in reported results.

Similar to most other interventions for musculoskeletal disorders, the reasons why the SSMP might induce an immediate change in symptoms are unclear, but it is apparent that the majority of patients in this study experienced significant within-session change in pain. This response might be secondary to soft tissue or joint displacement, changing sensory motor control, neuromodulation, contextual effects or for reasons yet to be determined. ${ }^{3}$ Along with further research relating to the reliability and predictive value of the SSMP, development of the understanding of the possible mechanism of action could shed further light on the applicability of this procedure for people reporting shoulder pain.

The importance of generating symptom modification based on the SSMP requires further evaluation. It is widely suggested that creating a change in symptoms within the treatment session is desirable. This study cannot provide a definitive answer to this hypothesis, but it is interesting to note that the extent of the change in patient self-report of pain was not well associated with combined pain and function scores, according to the SPADI, in the short term $(<1$ month) or mid-term ( $<3$ months). However, between-session changes were substantially associated with combined pain and function 
scores, according to the SPADI, over the first week, but beyond this short time frame the extent of the association diminished. This finding is similar to a previous shoulder study where between-session changes were not well associated with subsequent mid-term functional outcomes. ${ }^{9}$

\section{Limitations}

This study was conducted in one centre with a limited number of patients. Most of the physiotherapists involved in the study had not been formally trained in the SSMP and had varied experience using the procedure. However, this reflects real-world practice and also extends an approach reported previously. ${ }^{13}$

\section{Implications}

Based on this study, there is insufficient evidence to recommend the SSMP as a reliable or validated evidence tool for physical examination of patients with shoulder problems.

\section{CONCLUSION}

The SSMP has been proposed as an alternative to current approaches to examination. However, this study suggests moderate reliability of the SSMP, which might be regarded as insufficient to guide clinical decision-making. Furthermore, the importance of within-session changes as a basis for understanding future clinical outcome is uncertain, but between-session changes seem to be more strongly associated with clinical outcome in the short term but not the mid-term based on these data.

Acknowledgements The authors would like to thank all the outpatient MSK physiotherapists at the Spire Bushey Hospital who helped in the data collection of this study.

Contributors AM, SM, and CL designed the study protocol. AM collected the data. $\mathrm{CL}$ performed the statistical analysis. All authors contributed, read and approved the final manuscript.

Funding The authors have not declared a specific grant for this research from any funding agency in the public, commercial or not-for-profit sectors.

Competing interests $\mathrm{AM}$ and $\mathrm{CL}$ teach and lecture internationally on the assessment and management of shoulder pain

\section{Patient consent Obtained.}

Ethical approval information Ethical approval was obtained from the School of Health and Related Research, University of Sheffield, Research Ethics Committee (ref no: 006717).

Provenance and peer review Not commissioned; internally peer reviewed. Data sharing statement No additional data are available.

Open Access This is an Open Access article distributed in accordance with the Creative Commons Attribution Non Commercial (CC BY-NC 4.0) license, which permits others to distribute, remix, adapt, build upon this work non-commercially, and license their derivative works on different terms, provided the original work is properly cited and the use is non-commercial. See: http://creativecommons.org/ licenses/by-nc/4.0/ (c) Article author(s) (or their employer(s) unless otherwise stated in the text of the article) 2018. All rights reserved. No commercial use is permitted unless otherwise expressly granted.

\section{REFERENCES}

1. VosT, FlaxmanAD, NaghaviM, et al. Years lived with disability (YLDs) for 1160 sequelae of 289 diseases and injuries 1990-2010: a systematic analysis for the Global Burden of Disease Study 2010. Lancet 2012;380:2163-96.

2. OttenheijmRP, JooreMA, WalenkampGH, et al. The Maastricht Ultrasound Shoulder pain trial (MUST): ultrasound imaging as a diagnostic triage tool to improve management of patients with nonchronic shoulder pain in primary care. BMC Musculoskelet Disord 2011;12:154.

3. LewisJS. Rotator cuff tendinopathy/subacromial impingement syndrome: is it time for a new method of assessment? Br J Sports Med 2009;43:259-64.

4. LittlewoodC, MalliarasP, BatemanM, et al. The central nervous system - an additional consideration in 'rotator cuff tendinopathy' and a potential basis for understanding response to loaded therapeutic exercise. Man Ther 2013;18:468-72.

5. MayS, Chance-LarsenK, LittlewoodC, et al. Reliability of physical examination tests used in the assessment of patients with shoulder problems: a systematic review. Physiotherapy 2010;96:179-90.

6. HegedusEJ, GoodeAP, CookCE, et al. Which physical examination tests provide clinicians with the most value when examining the shoulder? Update of a systematic review with meta-analysis of individual tests. Br J Sports Med 2012;46:964-78.

7. HanchardNC, LenzaM, HandollHH, et al. Physical tests for shoulder impingements and local lesions of bursa, tendon or labrum that may accompany impingement. Cochrane Database Syst Rev 2013;30:CD007427.

8. CookC, LawrenceJ, MichalakK, et al. Is there preliminary value to a within- and/or between-session change for determining shortterm outcomes of manual therapy on mechanical neck pain? J Man Manip Ther 2014;22:173-80.

9. GarrisonJC, ShanleyE, ThigpenC, et al. Between-session changes predict overall perception of improvement but not functional improvement in patients with shoulder impingement syndrome seen for physical therapy: an observational study. Physiother Theory Pract 2011;27:137-45.

10. CookCE, ShowalterC, KabbazV, et al. Can a within/between-session change in pain during reassessment predict outcome using a manual therapy intervention in patients with mechanical low back pain? Man Ther 2012;17:325-9.

11. WrightAA, AbbottJH, BaxterD, et al. The ability of a sustained within-session finding of pain reduction during traction to dictate improved outcomes from a manual therapy approach on patients with osteoarthritis of the hip. J Man Manip Ther 2010;18:166-72.

12. BahatHS, KernerO. The Shoulder Symptom Modification Procedure (SSMP): a reliability study. J Nov Physiother 2016;s3.

13. LewisJS, McCreeshK, BarrattE, et al. Inter-rater reliability of the Shoulder Symptom Modification Procedure in people with shoulder pain. BMJ Open Sport Exerc Med 2016;2:e000181.

14. WilliamsJW, HollemanDR, SimelDL. Measuring shoulder function with the Shoulder Pain and Disability Index. J Rheumatol 1995;22:727-32.

15. MacDermidJC, SolomonP, PrkachinK. The Shoulder Pain and Disability Index demonstrates factor, construct and longitudinal validity. BMC Musculoskelet Disord 2006;7:12.

16. LandisJR, KochGG. The measurement of observer agreement for categorical data. Biometrics 1977;33:159-74.

17. SimJ, WrightCC. The kappa statistic in reliability studies: use, interpretation, and sample size requirements. Phys Ther 2005;85:257-68.

18. MayS, LittlewoodC, BishopA. Reliability of procedures used in the physical examination of non-specific low back pain: a systematic review. Aust J Physiother 2006;52:91-102. 